\title{
Ustekinumab for the treatment of refractory pediatric Crohn's disease: a single-center experience
}

\author{
Alexandra Cohen ${ }^{1}$, Najma Ahmed ${ }^{1,2}$, Ana Sant'Anna ${ }^{1,2}$ \\ ${ }^{I}$ McGill University, Montreal, QC; ${ }^{2}$ Division of Gastroenterology and Nutrition, Department of Pediatrics, Montreal Children's Hospital, \\ Montreal, QC, Canada
}

Background/Aims: Despite the well-established efficacy of tumor necrosis factor (TNF) antagonists as treatment options for Crohn's disease, many pediatric patients need a change in therapy due to adverse events and loss of response, highlighting the necessity for medications with a different mechanism of action. Ustekinumab has been shown to be effective in inducing clinical remission in some adults with disease refractory to anti-TNF agents, however, minimal data exists in the pediatric population. Methods: We conducted a retrospective chart review of 11 pediatric patients receiving ustekinumab, specifically extracting baseline data, information on prior treatment and response, indications for starting ustekinumab, clinical information, and laboratory parameters pre- and post-therapy. Clinical response was defined as a decrease in abbreviated Pediatric Crohn's Disease Activity Index score. Results: Patients ranged from 12 to 17 years of age upon initiation of treatment with ustekinumab. Five of 11 patients demonstrated a clinical response. Among these patients, 2 remained in clinical remission, while the remaining 3 experienced a secondary loss of response. The other 6 patients were primary nonresponders who either remained unwell or demonstrated slight clinical worsening. All patients who clinically responded to ustekinumab and had an initially elevated CRP experienced complete normalization of their values. Mucosal healing was seen on endoscopy in 1 responder, with 2 other patients showing endoscopic improvement. Conclusions: These results demonstrate for the first time that ustekinumab has the potential to induce not only clinical and biochemical remission, but also endoscopic improvement, in the pediatric population. Future research is needed to determine factors that influence response to therapy. (Intest Res 2021;19:217-224)

Key Words: Inflammatory bowel disease; Mucosal healing; Remission; Clinical improvement; Inflammatory markers

\section{INTRODUCTION}

Over the past decade, worldwide incidence of inflammatory bowel disease (IBD) has been increasing, including in the pediatric population. While IBD primarily affects young adults, $15 \%$ to $25 \%$ of cases occur in childhood. ${ }^{2,3}$ By 5 years post-diagnosis, $18 \%$ of pediatric IBD patients have been found to require surgical intervention. ${ }^{4}$ Despite the well-established efficacy of anti-tumor necrosis factor (TNF) therapies such as inf-

Received December 5, 2019. Revised January 26, 2020.

Accepted January 27, 2020.

Correspondence to Ana Sant'Anna, Division of Gastroenterology and

Nutrition, Department of Pediatrics, Montreal Children's Hospital, 1001 Decarie Boulevard, room B04.2443, Montreal, QC H4A 3J1, Canada. Tel: +1514-412-4474, Fax: +1-514-412-4392, E-mail: ana.santanna@mcgill.ca liximab and adalimumab, some patients need a change in therapy due to allergic reactions, adverse events, as well as primary and secondary loss of response, highlighting the necessity for therapies acting by different mechanisms. ${ }^{5}$

Ustekinumab, a monoclonal antibody targeting the p40 subunit of interleukin-12 and interleukin-23, has been approved to treat adults with active psoriatic arthritis or moderately to severely active Crohn's disease (CD), as well as to treat both adults and children aged 12 or older with moderate to severe plaque psoriasis. ${ }^{6}$

Thus far, in the adult CD population, ustekinumab has demonstrated variable clinical response rates ranging from under $40 \%$ to as high as $84 \% .{ }^{7.8}$ When successful, it has been shown to induce both clinical remission and mucosal healing, includ- 
ing in patients with refractory $\mathrm{CD}$ who have already received an anti-TNF. ${ }^{-15}$ At present, limited data exists on ustekinumab use in pediatric $\mathrm{CD}$, showing variable results. ${ }^{16-20}$

We retrospectively describe 11 adolescent patients who received ustekinumab at the Montreal Children's Hospital with the goal of assessing its efficacy in inducing clinical, biochemical, and endoscopic remission.

\section{METHODS}

A retrospective chart review was performed for all patients at the Montreal Children's Hospital with CD who received ustekinumab between December 2015 and July 2018. Baseline data, information on prior treatment and response, indications for starting ustekinumab, clinical information, and laboratory parameters pre- and post-therapy were abstracted. $\mathrm{Du}-$ ration of disease, location, and phenotype was recorded according to the Montreal classification. ${ }^{21}$ Response to therapy was assessed using laboratory markers, clinical data, and both endoscopy and pathology reports when available. Clinical response was defined as a decrease in score on the abbreviated Pediatric Crohn's Disease Activity Index (abbrPCDAI), a version of the PCDAI used in retrospective studies when all data required to calculate the full PCDAI score is not available. Specifically, the variables removed were: height, hematocrit, albumin, and erythrocyte sedimentation rate (ESR). ${ }^{22}$ ESR could not be utilized, as it is not part of standard evaluation at our center. The abbrPCDAI has been found to demonstrate sufficient correlation with the full PCDAI. ${ }^{23}$ Scores range from 0 (clinical remission) to 70 (severe, active disease), with the suggested cutoff points being < 10 (remission), 10 to 15 (mild disease), 16 to 25 (moderate disease), and $>25$ (severe disease) ${ }^{23}$ Patients who attained abbrPCDAI scores in the remission range $(<10)$ at any point throughout the course of their treatment were classified as responders, with the exception of 2 patients who had low abbrPCDAI scores but severe disease on endoscopy or magnetic resonance enterography (MRE) both before and after ustekinumab therapy. These 2 patients, alongside those with abbrPCDAI scores $\geq 10$, were classified as nonresponders.

Two patients were excluded from the data analysis. One had less than 6 months of follow-up data, and in another, there was a discrepancy between the biochemical markers (normal) and clinical symptoms, and thus it was not possible to accurately determine if the patient had active disease since endoscopy/imaging was not performed.
Certain patients' medical charts were reviewed independently for another study (Chavannes et al.). ${ }^{18}$ In the cases of these patients, the overlap predominantly consists of baseline data, with the current study including additional outcome variables and longer follow-up. This study was approved by the Research Ethics Board of the McGill University Health Centre (approval No. 2019-4728). This study is a retrospective study using medical record review and so informed consent was waived.

Table 1. Baseline Data

\begin{tabular}{|c|c|}
\hline Variable & Value \\
\hline Male sex & $8 / 11(72.7)$ \\
\hline Age at diagnosis (yr) & $11.4 \pm 4.5$ \\
\hline \multicolumn{2}{|l|}{ Montreal classification } \\
\hline \multicolumn{2}{|l|}{ Age at diagnosis } \\
\hline $\mathrm{A} 1$ (< $17 \mathrm{yr})$ & $11 / 11(100)$ \\
\hline \multicolumn{2}{|l|}{ Location of disease } \\
\hline L1 (small bowel) & 0/11 (0) \\
\hline L2 (colon) & $6 / 11(54.5)$ \\
\hline L3 (ileocolonic) & $1 / 11(9.1)$ \\
\hline L3 + L4 (diffuse) & $4 / 11(36.4)$ \\
\hline \multicolumn{2}{|l|}{ Disease behavior } \\
\hline B1 & 9/11 (81.8) \\
\hline B2 & 2/11 (18.2) \\
\hline Perianal disease & 2/11 (18.2) \\
\hline \multicolumn{2}{|l|}{ Previous anti-TNF use } \\
\hline Infliximab & $7 / 11(63.6)$ \\
\hline Adalimumab & $1 / 11(9.1)$ \\
\hline Infliximab \& adalimumab & $3 / 11(27.3)$ \\
\hline Age at start of ustekinumab (yr) & $15.3 \pm 1.8$ \\
\hline Concomitant immunosuppression & $4 / 11(36.4)$ \\
\hline Concomitant corticosteroids & $4 / 11(36.4)$ \\
\hline abbrPCDAl & $22.3 \pm 9.8$ \\
\hline $\mathrm{CRP}(\mathrm{mg} / \mathrm{L})$ & $17.1 \pm 33.6$ \\
\hline Hematocrit (L/L) & $0.4 \pm 0.1$ \\
\hline Albumin (g/L) & $40.4 \pm 6.2$ \\
\hline Weight (kg) & $57.0 \pm 22.8$ \\
\hline Height $(\mathrm{cm})^{a}$ & $126.3 \pm 56.1$ \\
\hline BMI $\left(\mathrm{kg} / \mathrm{m}^{2}\right)^{b}$ & $23.0 \pm 7.1$ \\
\hline
\end{tabular}

Values are presented as number/number (\%) or mean \pm standard deviation. a Four patients were excluded from this calculation due to unavailable data. ${ }^{b} \mathrm{BMI}$ was calculated using the 7 patients for whom both height and weight data were available.

TNF, tumor necrosis factor; abbrPCDAl, abbreviated Pediatric Crohn's Disease Activity Index; CRP, C-reactive protein; BMI, body mass index. 


\section{RESULTS}

\section{Baseline Characteristics}

While there were 13 patients who received ustekinumab at the Montreal Children's Hospital, 2 of them met exclusion criteria and were thus eliminated from the study. Baseline data on 11 patients who received ustekinumab is shown in Table 1. Seventy-three percent were male. Mean age at diagnosis was $11.4 \pm 4.5$ years and mean age at induction with ustekinumab was $15.3 \pm 1.8$ years. Patients began ustekinumab at $3.9 \pm 4.1$ years after diagnosis.

All patients were diagnosed with CD by endoscopy. Ten out of 11 patients (90.9\%) had small bowel imaging; 1 out of 10 (10.0\%) by video capsule and 9 out of $10(90.0 \%)$ by MRE. According to the Montreal classification, 6 out of 11 patients (54.5\%) had colonic disease (L2), 1 out of 11 (9.1\%) had ileocolonic disease (L3), and 4 out of 11 (36.4\%) had diffuse disease (ileocolonic and gastroduodenal; L3+L4). Two patients out of 11 (18.2\%) had stricturing disease (B2) and 2 out of 11 (18.2\%) had perianal involvement (B1p or B2p).

Prior to receiving ustekinumab, all patients had received at least one anti-TNF agent. In 7 out of 11 cases (63.6\%), the patient received infliximab. One out of 11 (9.1\%) received adalimumab and 3 out of 11 (27.3\%) received both. Reasons for terminating infliximab were either due to primary nonresponse (36.4\%), secondary loss of response (36.4\%), or due to unacceptable adverse effects (psoriasis or other skin reactions resistant to topical therapies; $18.2 \%$ ). All patients receiving adalimumab had their therapy changed due to a secondary

Table 2. Reason for Terminating Previous Anti-TNFs ${ }^{a}$

\begin{tabular}{lll}
\hline Patient No. & \multicolumn{1}{c}{ Infliximab } & \multicolumn{1}{c}{ Adalimumab } \\
\hline 1 & Unacceptable side effect & $N A^{b}$ \\
\hline 2 & $N A^{b}$ & Secondary loss of response \\
3 & Primary nonresponse & Secondary loss of response \\
4 & Unacceptable side effect & $N A^{b}$ \\
5 & Primary nonresponse & $N A^{b}$ \\
\hline 6 & Primary nonresponse & Secondary loss of response \\
7 & Secondary loss of response & $N A^{b}$ \\
\hline 8 & Primary nonresponse & $N A^{b}$ \\
9 & Secondary loss of response & $N A^{b}$ \\
\hline 11 & Secondary loss of response & $N A^{b}$ \\
\hline
\end{tabular}

${ }^{a}$ Immunogenicity data was not consistently available.

${ }^{b}$ Patient did not receive this medication.

TNF, tumor necrosis factor; NA, not available. loss of response (Table 2). Furthermore, 10 out of 11 patients (90.9\%) had previously failed an immunomodulator (methotrexate and/or azathioprine).

Prior to the availability of intravenous ustekinumab in Canada, 6 out of 11 patients (54.5\%) were induced at a dose of 90 mg subcutaneously at weeks 0,1 , and 2 . One patient was induced subcutaneously with $270 \mathrm{mg}$ at week 0 , followed by 180 $\mathrm{mg}$ at weeks 1 and 2 . All other patients were induced intravenously with doses ranging from $4.5-7.5 \mathrm{mg} / \mathrm{kg}$. Subsequently, all patients received maintenance doses of $90 \mathrm{mg}$ subcutaneously every 8 weeks. Nine out of 11 patients (81.8\%) later reduced their maintenance intervals to every 4 weeks due to ongoing symptoms.

At the time of induction, 4 out of 11 patients (36.4\%) were taking concomitant immunomodulators (methotrexate or azathioprine) and 4 out of 11 patients (36.4\%) were taking corticosteroids.

\section{Response to Ustekinumab Therapy}

Five patients (45.5\%) exhibited an initial clinical response (abbrPCDAI score decreasing to $<10$ ) to ustekinumab, of which 2 out of $5(40.0 \%)$ maintained this response across all available follow-up data (range, 6-22 months). Patients 3 through 5 developed a secondary loss of response between 11 and 19 months following induction. To optimize the effect of ustekinumab, these 3 patients had their treatment intervals shortened from 8 to 4 weeks within 2 to 12 months of treatment at the discretion of the attending physician. Upon decreasing their intervals, all 3 patients went into clinical remission within 1 to 4 months, maintaining low abbrPCDAI scores for an additional 3 to 6 months. No patients suffered from perianal disease or skin disease.

Clinical response, as measured by the abbrPCDAI, is shown in Fig. 1A. All ustekinumab responders had a mean score decrease of $21.3 \pm 8.5$ within the first 3 to 6 months of treatment The aforementioned secondary losses of response experienced by Patients 3-5 were accompanied by an average increase in abbrPCDAI score of $16.5 \pm 6.0$.

Ustekinumab responders also saw an average weight gain of $5.3 \pm 3.4 \mathrm{~kg}$ over the course of 4 to 12 months following initiation of treatment, with the exception of 1 patient (Patient 2), who lost weight intentionally (Fig. 2).

Two responders (Patients 4 and 5) were taking oral corticosteroids at the start of treatment. Both achieved a temporary steroid-free remission before ultimately experiencing a loss of response to ustekinumab. 
A

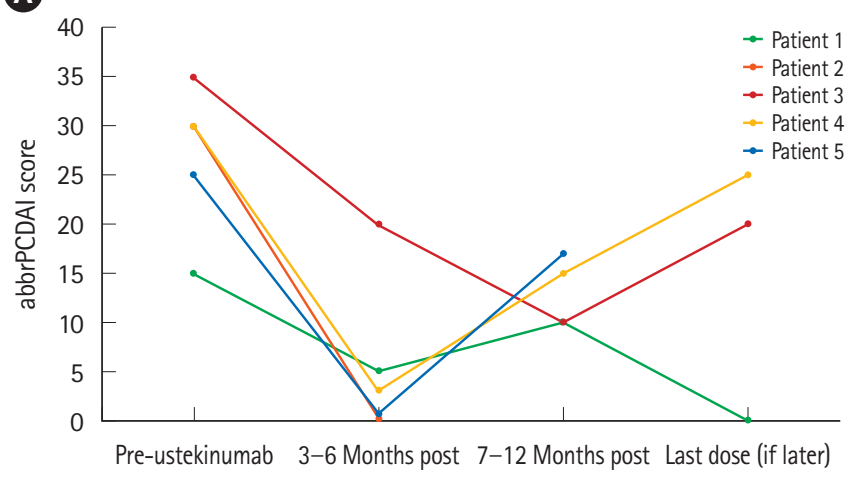

B

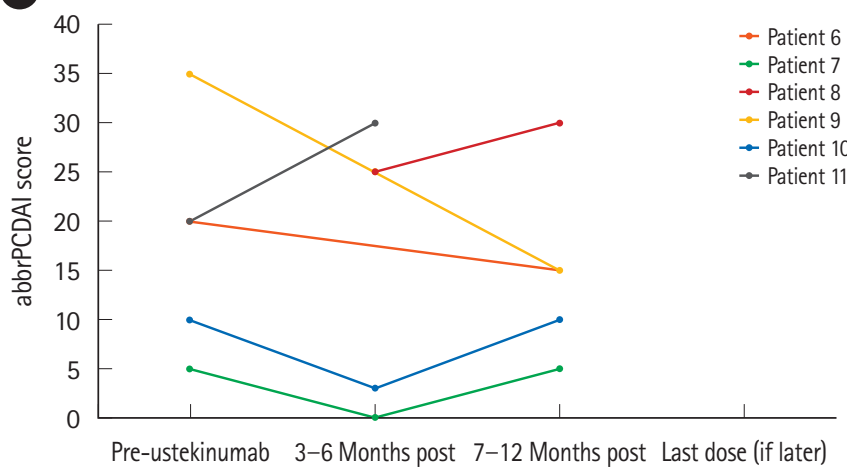

Fig. 1. Abbreviated Pediatric Crohn's Disease Activity Index (abbrPCDAI) scores over time. (A) Ustekinumab responders and (B) ustekinumab nonresponders. When more than one abbrPCDAl score was recorded in a given timeframe, all calculated scores were averaged together. Patients 7 and 10 were categorized as nonresponders despite low abbrPCDAl scores due to significant inflammation seen on imaging or endoscopy both before initiation of therapy and upon termination.

A

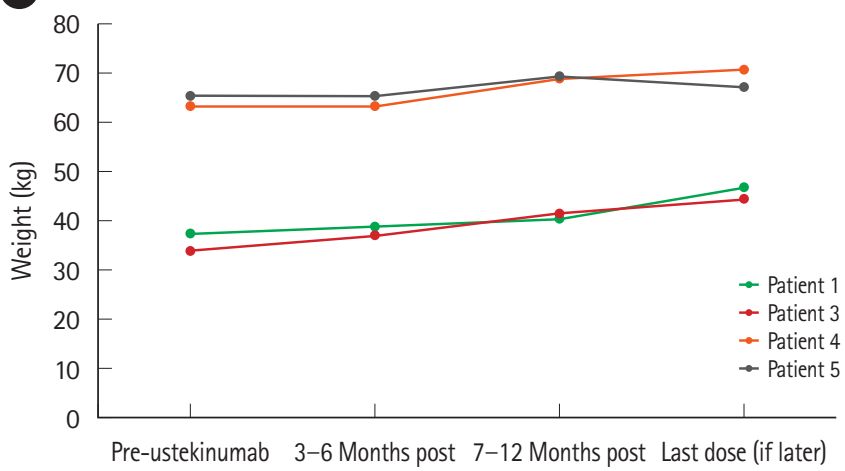

B

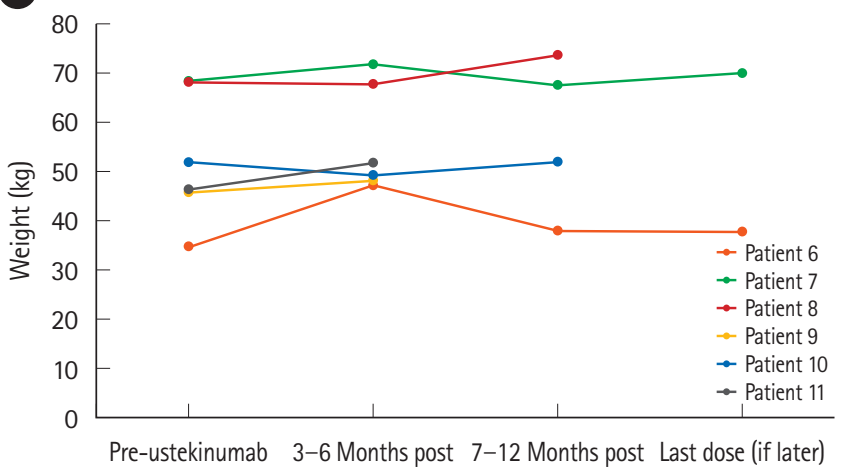

Fig. 2. Change in weight over the course of ustekinumab therapy. (A) Ustekinumab responders and (B) ustekinumab nonresponders. When more than one weight was recorded in a given timeframe, all calculated scores were averaged together. Patient 2 was excluded due to intentional weight loss over the course of ustekinumab therapy. Patients 4, 5, 9, and 11 were taking concomitant corticosteroids.

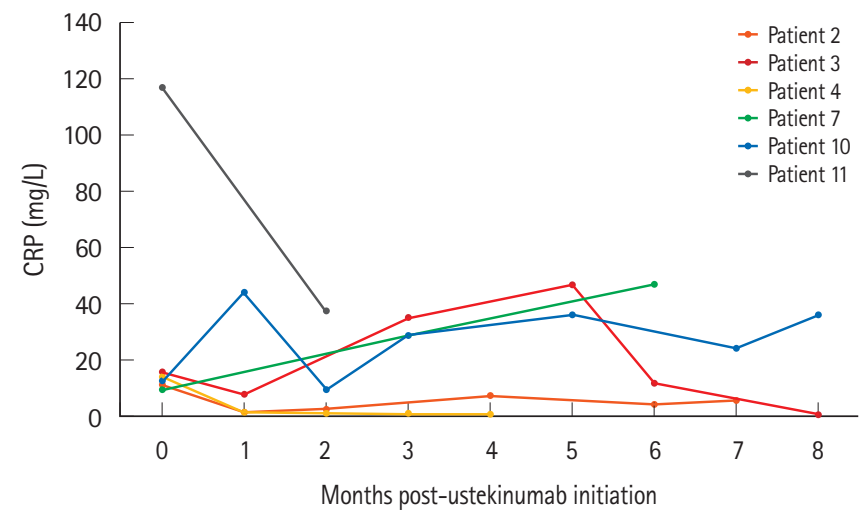

Fig. 3. Change in C-reactive protein (CRP) over the course of ustekinumab therapy. All patients with normal CRP values at baseline pre-ustekinumab despite elevated abbreviated Pediatric Crohn's Disease Activity Index scores were excluded from this graph.
The biochemical response of all patients was assessed using C-reactive protein (CRP) values both prior to initiating ustekinumab and throughout treatment (Fig. 3). Two out of 5 (40.0\%) had normal CRP values (range, $1.3-2.8 \mathrm{mg} / \mathrm{L}$ ) during a state of high disease activity (abbrPCDAI scores of 15 and 25 respectively) pre-induction, which therefore remained constant throughout treatment. Meanwhile, the values of Patients 2 and 4 normalized rapidly.

Only 1 ustekinumab responder had fecal calprotectin (FCP) measured both pre- and post-ustekinumab induction. Patient 5 began with a FCP of $384 \mu \mathrm{g} / \mathrm{g}$, which then increased to $>2,100 \mu \mathrm{g} / \mathrm{g}$ at 8 months post-induction, accompanied by a recurrence of clinical symptoms.

Endoscopic data pre- (maximum of 2 months before) and post-therapy was only available for 2 patients: Patient 2 , who 
had a sustained response to ustekinumab, and Patient 5, who experienced a secondary loss of response. Prior to initiating ustekinumab, Patient 2 had severe inflammation of the rectosigmoid and left colon. Eight months post-induction, however, Patient 2 was found to be in complete endoscopic remission. Patient 5 initially had severe left-sided colitis with moderately severe right-sided colitis, but at 7 months, a significant improvement was seen, with only mild findings on colonoscopy. No endoscopic data was collected following the loss of response.

\section{Nonresponse to Ustekinumab Therapy}

The remaining 6 patients (54.5\%; Patients 6-11) were ustekinumab primary nonresponders (follow-up range, 2-24 months). Patients 7 and 10 had low abbrPCDAI scores (Fig. 1B) at baseline and were categorized as nonresponders due to significant stricturing disease seen on MRE or endoscopy prior to initiating therapy that did not improve with treatment. Otherwise, none of these patients experienced a decrease in score significant enough to be qualified as remission-in fact, some scores increased as disease control worsened. The treatment interval was shortened from 8 to 4 weeks in all 6 cases with no success. No patients suffered from perianal disease and 1 patient ( $\mathrm{Pa}-$ tient 6) suffered from Henoch-Schonlein Purpura just prior to switching therapies.

Despite seeing no overall improvement in their symptom burden, the ustekinumab nonresponders, with the exception of Patient 10, were able to gain weight over the course of their treatment, which lasted on average $12.3 \pm 9.0$ months. All patients combined (excluding Patient 2 as was done previously) gained an average of $3.8 \pm 2.9 \mathrm{~kg}$.

Two nonresponders (Patients 9 and 11) were taking oral corticosteroids at the start of treatment. Both remained steroid dependent throughout treatment with ustekinumab.

Three patients (Patients 6, 8, and 9) did not have elevated CRP values even when symptomatic, with abbrPCDAI scores ranging from 20 to 35 . Patients 7 and 10 experienced fluctuating but consistently elevated CRPs. Conversely, Patient 11 did experience a significant decrease in CRP (from $117 \mathrm{mg} / \mathrm{L}$ preinduction to $37 \mathrm{mg} / \mathrm{L}$ at 2-month follow-up). Despite this partial biochemical improvement, he was switched to vedolizumab due to a deterioration in his clinical status.

Endoscopic data for nonresponders both pre- and post-induction with ustekinumab was only available for Patients 9 and 10. Patient 10 showed no signs of improvement on colonoscopy, with severe localized colitis in $10 \mathrm{~cm}$ of the sigmoid colon and luminal narrowing both before and after ustekinu-
Table 3. Ustekinumab Induction Mechanisms and Doses

\begin{tabular}{|c|c|c|c|c|}
\hline \multirow{2}{*}{$\begin{array}{c}\text { Patient } \\
\text { No. }\end{array}$} & \multicolumn{2}{|c|}{ IV ustekinumab } & \multicolumn{2}{|c|}{ SC ustekinumab } \\
\hline & $260 \mathrm{mg}$ & $520 \mathrm{mg}$ & $\begin{array}{c}90 \mathrm{mg} \\
\text { wk 0, 1, } 2\end{array}$ & $\begin{array}{c}270 \mathrm{mg} \text { wk } 0 \text {, } \\
180 \mathrm{mg} \text { wk } 1,2\end{array}$ \\
\hline 1 & & & $x$ & \\
\hline 2 & & $x$ & & \\
\hline 3 & & & $x$ & \\
\hline 4 & & & $x$ & \\
\hline 5 & & & & $x$ \\
\hline 6 & $x$ & & & \\
\hline 7 & & & $x$ & \\
\hline 8 & & & $x$ & \\
\hline 9 & $x$ & & & \\
\hline 10 & $x$ & & & \\
\hline 11 & & & $x$ & \\
\hline
\end{tabular}

IV, intravenous; SC, subcutaneous.

mab induction. Patient 9, despite a lack of clinical response, demonstrated initial endoscopic improvement, having severe colitis to the hepatic flexure prior to beginning treatment but then severe colitis in the rectum and mild colitis and in the descending and transverse colon 6 months later. However, when a colonoscopy was performed again at 8 months post-induction, the patient was found to have regressed to the same level of inflammation as was seen on the initial procedure. This finding, coupled with a persistently elevated FCP, resulted in the decision to terminate ustekinumab and begin vedolizumab.

\section{Safety Profile}

No patients in this study experienced any adverse events either during or following their treatment with ustekinumab. This safety was observed across a variety of induction mechanisms and doses (Table 3) as well as at treatment intervals of both 4 weeks and 8 weeks.

\section{DISCUSSION}

Two of 11 patients who had previously failed anti-TNF therapy were able to attain states of clinical remission within 2 to 7 months on ustekinumab, maintaining remission across 6 and 22 months of follow-up data respectively. Of the remaining 9 patients, 6 were primary nonresponders (defined as a lack of clinical improvement following induction therapy $)^{24}$ and 3 showed initial response with a subsequent secondary loss of response. 
Weight gain was seen in all but 1 patient, including those not labeled as having responded to ustekinumab. Notably, a confounding factor may be found in the fact that 2 out of 6 (33.3\%) of these nonresponders were taking oral corticosteroids at the time. Only 1 patient experienced significant clinical worsening while taking ustekinumab; patients generally either improved or maintained a consistent symptom burden, which may have allowed for the weight gain observed.

Assessing normalization of inflammatory markers can be a challenge, as it has been found that $20 \%$ to $25 \%$ of patients with CD do not exhibit increased CRP even when their disease is active due to a genetic polymorphism affecting CRP production. ${ }^{25}$ In our study, 5 patients did not have an elevated CRP prior to initiating ustekinumab treatment despite having elevated FCP (ranging from $384 \mu \mathrm{g} / \mathrm{g}$ to $>2,100 \mu \mathrm{g} / \mathrm{g}$ ). These patients have never had a documented elevated CRP. Of the clinical responders, 3 out of 5 had elevated CRP values prior to initiating ustekinumab therapy, all of which normalized within 1 month of clinical improvement. Even 1 patient who did not benefit from ustekinumab clinically experienced a rapid decrease in CRP within 2 months, thus demonstrating that ustekinumab is capable of improving inflammatory markers.

In the treatment of $\mathrm{CD}$, achieving mucosal healing is the ideal goal, as this has been associated with better outcomes, including a decrease in both corticosteroid use and hospitalizations. The International Organization for the Study of Inflammatory Bowel Diseases (IOIBD) defines mucosal healing as the absence of visible ulcerations and erosions on endoscopy. ${ }^{26}$ Sufficient endoscopic data was only available for 4 patients, 2 of whom responded to ustekinumab. Based on the IOIBD definition, ustekinumab did demonstrate the ability to induce mucosal healing in 1 of the patients who responded to therapy, also resulting in clear endoscopic improvement in both a responder and nonresponder. Ustekinumab has previously been found to cause mucosal healing in adults, ${ }^{11}$ but this has not yet been documented in the pediatric population.

Concomitant use of immunomodulators was previously found to be a potential predictive variable for clinical benefit of ustekinumab at 3 months, ${ }^{11}$ however we did not find any such relationship. Four of our patients were taking either methotrexate or azathioprine at the time of induction-one responded to ustekinumab and the other 3 did not. However, given the limited sample size a definite conclusion cannot be drawn regarding combination therapy.

Previous studies examining ustekinumab use in the pediatric population are few in number. Two case studies, docu- menting the clinical progress of 1 patient each with severe and refractory $\mathrm{CD}$, showed variable results, with ustekinumab inducing remission in 1 patient and failing in the other. ${ }^{17,20} \mathrm{~A}$ chart review examined the potential for clinical improvement on ustekinumab in 4 pediatric patients, 2 of whom experienced clinical worsening and 2 of whom demonstrated clinical improvement. ${ }^{16}$ Most recently, a multi-center cohort study examined 44 pediatric CD patients retrospectively, finding that at 12 months, $38.6 \%$ achieved clinical remission and $47.8 \%$ achieved clinical response. ${ }^{18}$ Another recent study found that amongst 52 children with either CD or ulcerative colitis initiating ustekinumab, $50 \%$ of those previously exposed to anti-TNF treatment were in steroid-free remission by week $52 .^{19}$

Our results demonstrate the potential for mucosal healing in pediatric patients receiving ustekinumab. Furthermore, while in the other pediatric studies, all patients received maintenance doses of ustekinumab every 7-8 weeks, doses in our study were shown to be given as frequently as every 4 weeks in an attempt to optimize therapy prior to labeling a patient as a nonresponder, with no adverse effects noted. Among the 8 patients who had their treatment interval shortened, 3 patients (37.5\%) showed a clinical response.

This data suggests that ustekinumab has the potential to result in clinical and endoscopic improvement, as well as decreased inflammatory markers. However, only 1 patient in this study was proven to maintain remission on ustekinumab for longer than 1 year, with this patient still clinically stable after 22 months of therapy. One responder only had 6 months of follow-up data available, while the others experienced secondary losses of response within 8-12 months of induction. Previous results have suggested with an even smaller sample size that the benefit of ustekinumab has not lasted longer than 15 months. ${ }^{20}$ Ideally, the next step will be to conduct a large prospective randomized control trial. Should this finding persist with a larger sample size, the next question will be whether anything can be done to optimize treatment and allow it to have a sustained effect.

This study was limited both by its small sample size, which decreased generalizability, and by the fact that it was retrospective in nature. Being retrospective, we were not able to control for certain differences amongst patients and were not able to collect all desired data at specific and regular time intervals. As a result, we did not have FCP and endoscopic data both pre- and post-ustekinumab for all patients, nor did we have the regular laboratory assessments needed to calculate 
the full PCDAI score. Furthermore, as described in Table 3, patients underwent several different induction protocols, which may have confounded the findings.

Future research also ought to investigate whether or not ustekinumab has a greater treatment effect in pediatric patients who are anti-TNF-naïve when compared to patients who have already failed TNF antagonist therapy, as has been found to be the case with vedolizumab. ${ }^{27}$ Data from the UNITI-2 study, with $69 \%$ of patients who were completely TNF antagonist-naïve, showed that these patients had higher rates of absolute response and remission than those who had previously failed anti-TNF therapies. ${ }^{28}$ Similarly, in our population that was not biologic-naïve, the response rate was much lower. However, by shortening the interval, some patients were able to achieve a clinical response.

Ustekinumab is a promising treatment option not only for adults with CD, but for children as well. Future research is needed to determine factors that influence response to therapy and which patients are most likely to benefit from this medication in order to optimize treatment strategies.

\section{ADDITIONAL INFORMATION}

\section{Funding Source}

The authors received no financial support for the research, authorship, and/or publication of this article.

\section{Conflict of Interest}

No potential conflict of interest relevant to this article was reported.

\section{Author Contribution}

Conceptualization: Cohen A, Ahmed N, Sant'Anna A. Formal analysis: Cohen A. Investigation: Cohen A. Methodology: Cohen A, Sant'Anna A. Project administration: Sant'Anna A. Resources: Sant'Anna A, Ahmed N. Supervision: Sant'Anna A. Visualization: Cohen A. Writing - original draft: Cohen A. Writing - review and editing: Cohen A, Ahmed N, Sant'Anna A. Approval of final manuscript: all authors.

\section{ORCID}

Cohen A

Ahmed N

https://orcid.org/0000-0001-9593-9093

Sant'Anna A

\section{REFERENCES}

1. Burisch J, Munkholm P. Inflammatory bowel disease epidemiology. Curr Opin Gastroenterol 2013;29:357-362.

2. Benchimol EI, Fortinsky KJ, Gozdyra P, van den Heuvel M, van Limbergen J, Griffiths AM. Epidemiology of pediatric inflammatory bowel disease: a systematic review of international trends. Inflamm Bowel Dis 2011;17:423-439.

3. Kim SC, Ferry GD. Inflammatory bowel diseases in pediatric and adolescent patients: clinical, therapeutic, and psychosocial considerations. Gastroenterology 2004;126:1550-1560.

4. Jakobsen C, Bartek J Jr, Wewer V, et al. Differences in phenotype and disease course in adult and paediatric inflammatory bowel disease: a population-based study. Aliment Pharmacol Ther 2011;34:1217-1224.

5. Hyams JS, Lerer T, Griffiths A, et al. Long-term outcome of maintenance infliximab therapy in children with Crohn's disease. Inflamm Bowel Dis 2009;15:816-822.

6. Janssen Inc. STELARA ${ }^{\circledR}$ product monograph [Internet]. [cited 2015 Dec 10]. https://www.janssen.com/products.

7. Khorrami S, Ginard D, Marín-Jiménez I, et al. Ustekinumab for the treatment of refractory Crohn's disease: the Spanish experience in a large multicentre open-label cohort. Inflamm Bowel Dis 2016;22:1662-1669.

8. Sandborn WJ, Gasink C, Gao LL, et al. Ustekinumab induction and maintenance therapy in refractory Crohn's disease. N Engl J Med 2012;367:1519-1528.

9. Sandborn WJ, Feagan BG, Fedorak RN, et al. A randomized trial of ustekinumab, a human interleukin-12/23 monoclonal antibody, in patients with moderate-to-severe Crohn's disease. Gastroenterology 2008;135:1130-1141.

10. Kopylov U, Afif W, Cohen A, et al. Subcutaneous ustekinumab for the treatment of anti-TNF resistant Crohn's disease: the McGill experience. J Crohns Colitis 2014;8:1516-1522.

11. Wils P, Bouhnik Y, Michetti P, et al. Subcutaneous ustekinumab provides clinical benefit for two-thirds of patients with Crohn's disease refractory to anti-tumor necrosis factor agents. Clin Gastroenterol Hepatol 2016;14:242-250.

12. Harris KA, Horst S, Gadani A, et al. Patients with refractory Crohn's disease successfully treated with ustekinumab. Inflamm Bowel Dis 2016;22:397-401.

13. Tuskey A, Behm BW. Profile of ustekinumab and its potential in patients with moderate-to-severe Crohn's disease. Clin Exp Gastroenterol 2014;7:173-179.

14. Scherl EJ, Kumar S, Warren RU. Review of the safety and efficacy of ustekinumab. Therap Adv Gastroenterol 2010;3:321- 
328.

15. Lamb YN, Duggan ST. Ustekinumab: a review in moderate to severe Crohn's disease. Drugs 2017;77:1105-1114.

16. Bishop C, Simon H, Suskind D, Lee D, Wahbeh G. Ustekinum$\mathrm{ab}$ in pediatric Crohn disease patients. J Pediatr Gastroenterol Nutr 2016;63:348-351.

17. Cameron FL, Garrick V, Russell RK. Ustekinumab in treatment of refractory paediatric Crohn disease. J Pediatr Gastroenterol Nutr 2016;62:e30.

18. Chavannes M, Martinez-Vinson C, Hart L, et al. Management of paediatric patients with medically refractory Crohn's disease using ustekinumab: a multi-centred cohort study. J Crohns Colitis 2019;13:578-584.

19. Dayan JR, Dolinger M, Benkov K, et al. Real world experience with ustekinumab in children and young adults at a tertiary care pediatric inflammatory bowel disease center. J Pediatr Gastroenterol Nutr 2019;69:61-67.

20. Rinawi F, Rosenbach Y, Assa A, Shamir R. Ustekinumab for resistant pediatric Crohn disease. J Pediatr Gastroenterol Nutr 2016;62:e34-e35.

21. Satsangi J, Silverberg MS, Vermeire S, Colombel JF. The Montreal classification of inflammatory bowel disease: controversies, consensus, and implications. Gut 2006;55:749-753.

22. Shepanski MA, Markowitz JE, Mamula P, Hurd LB, Baldassa- no RN. Is an abbreviated pediatric Crohn's disease activity index better than the original? J Pediatr Gastroenterol Nutr 2004;39:68-72.

23. Turner D, Griffiths AM, Walters TD, et al. Mathematical weighting of the pediatric Crohn's disease activity index (PCDAI) and comparison with its other short versions. Inflamm Bowel Dis 2012;18:55-62.

24. Roda G, Jharap B, Neeraj N, Colombel JF. Loss of response to anti-TNFs: definition, epidemiology, and management. Clin Transl Gastroenterol 2016;7:e135.

25. Jones J, Loftus EV Jr, Panaccione R, et al. Relationships between disease activity and serum and fecal biomarkers in patients with Crohn's disease. Clin Gastroenterol Hepatol 2008; 6:1218-1224.

26. Dave M, Loftus EV Jr. Mucosal healing in inflammatory bowel disease-a true paradigm of success? Gastroenterol Hepatol (NY) 2012;8:29-38.

27. Sands BE, Sandborn WJ, Van Assche G, et al. Vedolizumab as induction and maintenance therapy for Crohn's disease in patients naïve to or who have failed tumor necrosis factor antagonist therapy. Inflamm Bowel Dis 2017;23:97-106.

28. Feagan BG, Sandborn WJ, Gasink C, et al. Ustekinumab as induction and maintenance therapy for Crohn's disease. N Engl J Med 2016;375:1946-1960. 